\title{
LAGRANGIAN APPROXIMATIONS AND WEAK SOLUTIONS OF THE NAVIER-STOKES EQUATIONS
}

\author{
WERNER VARNHORN \\ Faculty of Mathematics, University of Kassel \\ Heinrich-Plett-Str. 40, 34132 Kassel, Germany \\ E-mail:varnhorn@mathematik.uni-kassel.de
}

\begin{abstract}
The motion of a viscous incompressible fluid flow in bounded domains with a smooth boundary can be described by the nonlinear Navier-Stokes equations. This description corresponds to the so-called Eulerian approach. We develop a new approximation method for the Navier-Stokes equations in both the stationary and the non-stationary case by a suitable coupling of the Eulerian and the Lagrangian representation of the flow, where the latter is defined by the trajectories of the particles of the fluid. The method leads to a sequence of uniquely determined approximate solutions with a high degree of regularity containing a convergent subsequence with limit function $v$ such that $v$ is a weak solution of the Navier-Stokes equations.
\end{abstract}

0. Introduction, notation and main results. We consider the non-stationary nonlinear Navier-Stokes equations

$$
\begin{aligned}
\partial_{t} v-\nu \Delta v+v \cdot \nabla v+\nabla p & =F & & \text { in } G_{T}, \\
\nabla \cdot v & =0 & & \text { in } G_{T}, \\
v & =0 & & \text { on } \partial G, \\
v & =v_{0} & & \text { for } t=0 .
\end{aligned}
$$

Here $G_{T}:=(0, T) \times G$ is a bounded cylindrical domain, where $T>0$ and $G \subset \mathbb{R}^{3}$ has a smooth boundary $\partial G$. These equations describe the motion of a viscous incompressible time dependent fluid confined to $G$ for $0<t<T$. Here $v=v(t, x)=$ $\left(v_{1}(t, x), v_{2}(t, x), v_{3}(t, x)\right)$ represents the velocity and $p=p(t, x)$ the kinematic pressure at time $t$ at position $x \in G$. The constant $\nu>0$ (kinematic viscosity), the external force density $F$, and the initial velocity $v_{0}$ are given data.

Besides the description of a flow by its velocity $v$ and pressure $p$ there is another approach using the Lagrangian coordinates $X\left(t, s, x_{s}\right) \in G,[2]$. Here the function

2000 Mathematics Subject Classification: Primary 35B65; Secondary 67D05.

Key words and phrases: Navier-Stokes equation, Lagrangian approximation, weak solution. The paper is in final form and no version of it will be published elsewhere. 


$$
t \rightarrow x(t)=X\left(t, s, x_{s}\right)
$$

denotes the trajectory of a fluid particle, which at initial time $t=s$ is located at $x_{s} \in G$. This approach has been used for the treatment of the Navier-Stokes and the transport equations ([4], [7], [12], [14]) and is of great importance for the numerical computation of a flow involving different media with interfaces. In general, both representations are related by the equations

$$
\dot{x}(t)=v(t, x(t)), \quad x(s)=x_{s} \in G,
$$

which is an initial value problem for ordinary differential equations if the velocity $v$ is known.

Besides the non-stationary Navier-Stokes equations mentioned above we also consider the stationary system

$$
\begin{aligned}
-\nu \Delta v+v \cdot \nabla v+\nabla p & =F & & \text { in } G, \\
\nabla \cdot v & =0 & & \text { in } G, \\
v & =0 & & \text { on } \partial G .
\end{aligned}
$$

In this case all functions do not depend on time: The functions

$$
v=v(x)=\left(v_{1}(x), v_{2}(x), v_{3}(x)\right), \quad p=p(x)
$$

denote the unknown velocity and pressure, respectively. Because for steady flow the streamlines and the trajectories of the fluid particles coincide, both approaches mentioned above are related by the autonomous system of ordinary differential equations

$$
\dot{x}(t)=v(x(t)), \quad x(0)=x_{0} \in G,
$$

which is an initial value problem for

$$
t \rightarrow x(t)=X\left(t, 0, x_{0}\right)=X\left(t, x_{0}\right)
$$

if the velocity field $v$ is known.

In the present paper we construct an energy conserving Lagrangian difference quotient, which approximates the nonlinear convective term $v \cdot \nabla v$ in (1) and (3). By a suitable time delay in the non-stationary case it is possible to determine the trajectories of the fluid particles from the velocity field and vice versa successively, such that the resulting equations can be solved for all time. A special initial construction of compatible data ensures, that the corresponding solution is uniquely determined and has a high degree of regularity uniformly in time. Passing to the limit for the Lagrangian difference quotient the following convergence result is shown: There always exists a subsequence of the solutions, which for all time converges to a weak solution of (1) and (3), respectively.

Let us outline our notation: We set $\mathbb{N}_{0}:=\mathbb{N} \cup\{0\}$. The set $I \subset \mathbb{R}$ always denotes a compact interval and $G \subset \mathbb{R}^{3}$ a bounded domain with smooth boundary $\partial G$ and closure $\bar{G}:=G \cup \partial G$. Throughout the paper we use the same symbols for scalar and vector valued functions as well as for the corresponding function spaces and norms.

We need the spaces $L^{p}(G)(1 \leq p \leq \infty)$ of integrable functions and the spaces $C^{m}(G)$, $C^{m}(\bar{G}), C_{0}^{m}(G)$ of continuous functions. Here the subscript 0 denotes a compact support 
in $G$. Moreover, we need the usual Sobolev (Hilbert) spaces $H^{m}(G), H_{0}^{m}(G)\left(m \in \mathbb{N}_{0}\right)$, where in particular $H_{0}^{0}(G)=H^{0}(G)=L^{2}(G)$.

We also use the Banach-valued spaces $C^{m}(I, B)\left(m \in \mathbb{N}_{0}\right)$ and $L^{p}(a, b, B)$, where $a, b \in \mathbb{R}(a<b)$ and $B$ is any of the spaces above. Instead of $C^{0}(\cdot)$ we write $C(\cdot)$. The norm in $L^{p}(G)$ and in $H^{m}(G)$ is denoted by $\|\cdot\|_{0, p}$ and $\|\cdot\|_{m}$, respectively, where in particular we set

$$
\|\cdot\|:=\|\cdot\|_{0,2}=\|\cdot\|_{0}, \quad\|\cdot\|_{\infty}:=\|\cdot\|_{0, \infty}:=\underset{x \in G}{\operatorname{ess} \sup }|\cdot(x)|
$$

with the Euclidian norm $|\cdot|$. For $v=\left(v_{1}, v_{2}, v_{3}\right)$ and $u=\left(u_{1}, u_{2}, u_{3}\right)$ we use

$$
(v, u):=\int_{G} \sum_{i=1}^{3} v_{i}(x) u_{i}(x) d x
$$

as scalar product in $L^{2}(G)$. By $H(G)$ and $V(G)$ we denote the closure of

$$
D(G):=\left\{u \in C_{0}^{\infty}(G) \mid \nabla \cdot u=0\right\}
$$

in $L^{2}(G)$ and $H^{1}(G)$, respectively, i.e. we use the notation

$$
\begin{aligned}
H(G) & :=\overline{D(G)}^{\|\cdot\|}, \\
V(G) & :=\overline{D(G)}^{\|\cdot\|_{1}} .
\end{aligned}
$$

Here, as usual, $C_{0}^{\infty}(G)$ denotes the space of all $C^{\infty}(G)$-functions with compact support confined to $G$.

The operator

$$
P: L^{2}(G) \rightarrow H(G)
$$

denotes the orthogonal projector associated with the orthogonal decomposition

$$
L^{2}(G)=H(G) \oplus\left\{u \in L^{2}(G) \mid u=\nabla p, p \in H^{1}(G)\right\} .
$$

With $\partial_{i}(i=1,2,3)$ as the partial derivative with respect to $x_{i}$ we set $\nabla:=\left(\partial_{1}, \partial_{2}, \partial_{3}\right)=$ grad and define

$$
\nabla \cdot v:=\sum_{i=1}^{3} \partial_{i} v_{i}, \quad v \cdot \nabla u:=\left(\sum_{i=1}^{3} v_{i} \partial_{i} u_{j}\right)_{j}, \quad \nabla v:=\left(\partial_{j} v_{k}\right)_{k j}
$$

In $V(G)$ and $H_{0}^{1}(G)$ we mostly use

$$
(\nabla v, \nabla u):=\sum_{j=1}^{3}\left(\partial_{j} v, \partial_{j} u\right)=\sum_{i, j=1}^{3}\left(\partial_{j} v_{i}, \partial_{j} u_{i}\right)
$$

and $\|\nabla v\|:=(\nabla v, \nabla v)^{\frac{1}{2}}$ as scalar product and norm, respectively, since $G$ is bounded.

1. The stationary Navier-Stokes equations. Let us start by recalling some facts, which concern existence and uniqueness for the solution of the initial value problem (4): If the velocity field $v$ belongs to the space $C^{1,0}(\bar{G})$, defined by

$$
C^{1,0}(\bar{G}):=\left\{u \in C^{1}(\bar{G}) \mid u=0 \text { on } \partial G\right\},
$$

then for all $x_{0} \in G$ the solution

$$
t \rightarrow x(t)=X\left(t, x_{0}\right)
$$


of (4) is uniquely determined and exists for all $t \in \mathbb{R}$. Here the global existence follows from the fact that $v=0$ on the boundary $\partial G$ implies that the trajectories remain in $G$ for all times. Due to the uniqueness, the set of mappings

$$
\Re=\{X(t, \cdot): G \rightarrow G \mid t \in \mathbb{R}\}
$$

defines a commutative group of $C^{1}$-diffeomorphisms in $G$. In particular, for $t \in \mathbb{R}$ the inverse mapping $X(t, \cdot)^{-1}$ of $X(t, \cdot)$ is given by $X(-t, \cdot)$, i.e.

$$
X(t, \cdot) \circ X(-t, \cdot):=X(t, X(-t, \cdot))=X(t-t, \cdot)=X(0, \cdot)=\mathrm{id},
$$

or, equivalently,

$$
X(t, X(-t, x))=x
$$

for all $t \in \mathbb{R}$ and $x \in G$. Moreover, we obtain $\operatorname{det} \nabla X(t, x)=1$ if additionally

$$
v \in C_{\sigma}^{1,0}(\bar{G})=\left\{u \in C^{1,0}(\bar{G}) \mid \nabla \cdot u=0\right\} .
$$

This important measure preserving property implies

$$
(f, g)=(f \circ X(t, \cdot), g \circ X(t, \cdot))
$$

for all functions $f, g \in L^{2}(G)$ as well as

$$
\|f\|_{0, p}=\|f \circ X(t, \cdot)\|_{0, p}, \quad f \in L^{p}(G), \quad 1 \leq p \leq \infty .
$$

Next let us consider the Navier-Stokes boundary value problem (3). It is well known that, given $F \in L^{2}(G)$, there is at least one function $v$ satisfying (3) in a weak sense. Let us recall:

Definition 1.1. Let $F \in L^{2}(G)$ be given. A function $v \in V(G)$ satisfying for all $\Phi \in D(G)$ the identity

$$
\nu(\nabla v, \nabla \Phi)-(v \cdot \nabla \Phi, v)=(F, \Phi)
$$

is called a weak solution of the Navier-Stokes equations (3), and (7) is called the weak form of $(3)$.

For a suitable approximation of the nonlinear term $v \cdot \nabla v$ let us keep in mind its physical deduction. It is a convective term arising from the total or substantial derivative of the velocity vector $v$. Thus it seems to be reasonable to use a total difference quotient for its approximation.

To do so, let $v \in C_{\sigma}^{1,0}(\bar{G})$ be given. Then for any $\varepsilon \in \mathbb{R}$ the mapping $X(\varepsilon, \cdot): G \rightarrow G$ and its inverse $X(-\varepsilon, \cdot)$ are well defined. Consider for some $u \in C^{1}(G)$ and $x \in G$ the one-sided Lagrangian difference quotients

$$
L_{+}^{\varepsilon} u(x)=\frac{1}{\varepsilon}[u(X(\varepsilon, \cdot))-u(x)], \quad L_{-}^{\varepsilon} u(x)=\frac{1}{\varepsilon}[u(x)-u(X(-\varepsilon, \cdot))],
$$

and the central Lagrangian difference quotient

$$
L^{\varepsilon} u(x)=\frac{1}{2}\left(L_{+}^{\varepsilon} u(x)+L_{-}^{\varepsilon} u(x)\right) .
$$

Since for sufficiently regular functions we find

$$
L_{-}^{\varepsilon} u(x) \rightarrow v(x) \cdot \nabla u(x) \quad \text { and } \quad L_{+}^{\varepsilon} u(x) \rightarrow v(x) \cdot \nabla u(x)
$$


as $\varepsilon \rightarrow 0$, all the above quotients can be used for the approximation of the term $v \cdot \nabla u$. There is, however, an important advantage of the central quotient (8) with respect to the conservation of the energy:

Lemma 1.2. Let $v \in C_{\sigma}^{1,0}(\bar{G})$ and $u, w \in L^{2}(G)$. Let $X(\varepsilon, \cdot)$ and $X(-\varepsilon, \cdot)$ denote the mappings constructed from the solution of (4). Then for the central quotient $L^{\varepsilon} u$ defined by (8) we have

$$
\left(L^{\varepsilon} u, w\right)=-\left(u, L^{\varepsilon} w\right)
$$

hence

$$
\left(L^{\varepsilon} u, u\right)=0 \text {. }
$$

Proof. Due to $v \in C_{\sigma}^{1,0}(\bar{G})$ the mappings $X(t, \cdot)$ and $X(-\varepsilon, \cdot)$ are measure preserving. Setting $X:=X(\varepsilon, \cdot)$, this implies

$$
\begin{aligned}
\left(L^{\varepsilon} u, w\right) & =\frac{1}{2 \varepsilon}\left(u \circ X-u \circ X^{-1}, w\right)=\frac{1}{2 \varepsilon}\left[(u \circ X, w)-\left(u \circ X^{-1}, w\right)\right] \\
& =\frac{1}{2 \varepsilon}\left[\left(u, w \circ X^{-1}\right)-(u, w \circ X)\right]=-\frac{1}{2 \varepsilon}\left[\left(u, w \circ X-w \circ X^{-1}\right)\right]=-\left(u, L^{\varepsilon} w\right) .
\end{aligned}
$$

The second assertion follows by setting $u=w$.

Let us point out that (9) is a Lagrangian analogy to the (Eulerian) relation

$$
(v \cdot \nabla u, w)=-(v \cdot \nabla w, u),
$$

valid for all functions $v \in V(G)$ and $u, w \in H_{0}^{1}(G)$, which analogously implies

$$
(v \cdot \nabla u, u)=0
$$

for all $v \in V(G), u \in H_{0}^{1}(G)$.

To establish an approximation procedure we assume that some approximate velocity field $v^{n}$ has already been found. To construct $v^{n+1}$ we proceed as follows:

1) Construct $X^{n}:=X\left(\frac{1}{n}, \cdot\right)$ and its inverse $X^{-n}:=X\left(-\frac{1}{n}, \cdot\right)$ from the initial value problem

$$
\dot{x}(t)=v^{n}(x(t)), \quad x(0)=x_{0} \in G .
$$

2) Construct $v^{n+1}$ and $p^{n+1}$ from the boundary value problem

$$
\begin{array}{rlrl}
-\nu \Delta v^{n+1}+\frac{n}{2}\left[v^{n+1} \circ X^{n}-v^{n+1} \circ X^{-n}\right]+\nabla p^{n+1} & =F & & \text { in } G, \\
\nabla \cdot v^{n+1}=0 & \text { in } G, \\
v^{n+1}=0 & \text { on } \partial G .
\end{array}
$$

The main result is now stated in the following

TheOREM 1.3. a) Assume $v^{n} \in H^{3}(G) \cap V(G)$ and $F \in H^{1}(G)$. Then for all $x_{0} \in G$ the initial value problem (13) is uniquely solvable, and the mappings

$$
X^{n}: G \rightarrow G, \quad X^{-n}: G \rightarrow G
$$

are measure preserving $C^{1}$-diffeomorphisms in $G$.

b) Moreover, there is a uniquely determined solution

$$
v^{n+1} \in H^{3}(G) \cap V(G), \nabla p^{n+1} \in H^{1}(G)
$$


of the equations (14). The velocity field $v^{n+1}$ satisfies the energy equation

$$
\nu\left\|\nabla v^{n+1}\right\|^{2}=\left(F, v^{n+1}\right) .
$$

c) Assume $v^{0} \in H^{3}(G) \cap V(G)$ and $F \in H^{1}(G)$. Let $\left(v^{n}\right)_{n}$ denote the sequence of unique solutions constructed in view of Part b). Then $\left(v^{n}\right)_{n}$ is bounded in $V(G)$, i.e.

$$
\left\|\nabla v^{n}\right\|^{2} \leq C_{G, F, \nu}
$$

for all $n \in \mathbb{N}$, where the constant $C_{G, F, \nu}$ does not depend on $n$.

d) The sequence $\left(v^{n}\right)_{n}$ has an accumulation point $v \in V(G)$ satisfying (7), i.e. $v$ is a weak solution of the Navier-Stokes equations (3).

Proof. 1.3a) Because $H^{3}(G)$ is continuously imbedded in $C^{1}(\bar{G})$ [1] and thus we have $H^{3}(G) \cap V(G) \subset C_{\sigma}^{1,0}(\bar{G})$, the initial value problem (13) is uniquely solvable, and $X^{n}$ as well as $X^{-n}$ have the asserted properties.

1.3b) Consider now the boundary value problem (14). By means of a Galerkin method (compare $[10,16]$ ) we can prove the existence of some function $v^{n+1} \in V(G) \subset H^{1}(G)$ satisfying the weak version of (14), i.e.

$$
\nu\left(\nabla v^{n+1}, \nabla \Phi\right)+\frac{n}{2}\left(v^{n+1} \circ X^{n}-v^{n+1} \circ X^{-n}, \Phi\right)=(F, \Phi)
$$

for all $\Phi \in D(G)$. Moreover, there is exactly one such function, because for the difference $w^{n+1}$ of two solutions we have

$$
\nu\left(\nabla w^{n+1}, \nabla w^{n+1}\right)=-\frac{n}{2}\left(w^{n+1} \circ X^{n}-w^{n+1} \circ X^{-n}, w^{n+1}\right)=0,
$$

hence $\left\|\nabla w^{n+1}\right\|=0$ and thus $w^{n+1}=0$ in $\bar{G}$ due to $w^{n+1}=0$ on $\partial G$. It remains to prove the regularity property $v^{n+1} \in H^{3}(G)$ if $F \in H^{1}(G)$. To do so we write (14) in the form of a linear Stokes system:

$$
\begin{array}{rlrl}
-\nu \Delta v^{n+1}+\nabla p^{n+1} & =K & & \text { in } G, \\
\nabla \cdot v^{n+1}=0 & & \text { in } G, \\
v^{n+1} & =0 & & \text { on } \partial G,
\end{array}
$$

where

$$
K=F-\frac{n}{2}\left[v^{n+1} \circ X^{n}-v^{n+1} \circ X^{-n}\right] .
$$

Then, using Cattabriga's estimate [3], we obtain $v^{n+1} \in H^{3}(G)$ if only $K \in H^{1}(G)$. Thus it suffices to show $v^{n+1} \circ X^{n} \in H^{1}(G)\left(v^{n+1} \circ X^{-n}\right.$ analogously). Because

$$
\left\|v^{n+1} \circ X^{n}\right\|=\left\|v^{n+1}\right\|<\infty
$$

since $v^{n+1} \in L^{2}(G)$, it remains to estimate the norm $\left\|\nabla\left(v^{n+1} \circ X^{n}\right)\right\|$.

To do so, setting $\varepsilon:=\frac{1}{n}$, we first observe

$$
\left\|\nabla\left(v^{n+1} \circ X^{n}\right)\right\| \leq\left\|\nabla_{\mathrm{x}} v^{n+1}(X(\varepsilon, \cdot))\right\|\|\nabla X(\varepsilon, \cdot)\|_{\infty}=\left\|\nabla v^{n+1}\right\|\left\|\nabla X^{n}\right\|_{\infty},
$$

and then

$$
\begin{aligned}
\frac{d}{d t}\left\|\nabla X^{n}\right\|_{\infty} & =\frac{d}{d t}\left\|\nabla X(t, \cdot)_{\mid t=\varepsilon}\right\|_{\infty} \leq\left\|\partial_{t} \nabla X(t, \cdot)_{\mid t=\varepsilon}\right\|_{\infty} \\
& =\left\|\nabla\left(v^{n}(X(t, \cdot))\right)_{\mid t=\varepsilon}\right\|_{\infty} \leq\left\|\nabla_{\mathrm{x}} v^{n} \circ X(\varepsilon, \cdot)\right\|_{\infty}\|\nabla X(\varepsilon, \cdot)\|_{\infty} \\
& =\left\|\nabla v^{n}\right\|_{\infty}\left\|\nabla X^{n}\right\|_{\infty},
\end{aligned}
$$


where $\left\|\nabla v^{n}\right\|_{\infty} \leq c\left\|v^{n}\right\|_{3}$ due to Sobolev's imbedding theorem [1]. The estimate (17) is a differential inequality for $\|\nabla X(t, \cdot)\|_{\infty}$ with initial value $\|\nabla X(0, \cdot)\|_{\infty}=\|I\|_{\infty}=1$. Thus, using Gronwall's Lemma, we find

$$
\left\|\nabla X^{n}\right\|_{\infty} \leq \exp \left(c \varepsilon\left\|v^{n}\right\|_{3}\right)<\infty
$$

and the first assertion is proved. The second assertion, i.e. the energy equation (15), follows from (16) with $\Phi=v^{n+1}$ using a density argument due to the orthogonality relation (10). This proves Part b) of Theorem 1.3.

1.3c) The boundedness of the above constructed sequence $\left(v^{n}\right)_{n}$ in $V(G)$ obviously follows from the energy equation (15) using

$$
\nu\left\|\nabla v^{n+1}\right\|^{2} \leq\|F\|\left\|v^{n+1}\right\| \leq c_{G}\|F\|\left\|\nabla v^{n+1}\right\|
$$

with the Poincaré constant $c_{G}$.

1.3d) Because $\left(v^{n}\right)_{n}$ is bounded in $V(G)$, there is a convergent subsequence, in the following again denoted by $\left(v^{n}\right)_{n}$, with limit $v \in V(G)$ such that $v^{n} \rightarrow v$ weakly as $n \rightarrow \infty$ with respect to the Dirichlet norm $\|\nabla \cdot\|$. Because the imbedding

$$
V(G) \subset L^{2}(G)
$$

is compact [1] we can again extract a subsequence such that, in addition, $v^{n} \rightarrow v$ strongly in $L^{2}(G)$ as $n \rightarrow \infty$, hence we have for all $\Phi \in D(G)$

$$
\left(\nabla\left(v^{n}-v\right), \nabla \Phi\right) \rightarrow 0 \quad \text { as } \quad n \rightarrow \infty
$$

and

$$
\left\|v^{n}-v\right\| \rightarrow 0 \quad \text { as } \quad n \rightarrow \infty .
$$

Thus $v$ is a weak solution of (3), if (18) and (19) are sufficient to proceed to the limit $n \rightarrow \infty$ also in the convective term. Hence using the orthogonality relations (9), (11) it remains to prove

$$
\frac{n}{2}\left(\Psi_{i} \circ X^{n}-\Psi_{i} \circ X^{-n}, v^{n+1}\right) \stackrel{n \rightarrow \infty}{\longrightarrow}\left(v \cdot \nabla \Psi_{i}, v\right),
$$

where

$$
\left\{\Psi_{i} \mid i \in \mathbb{N}\right\} \subset D(G)
$$

is a complete orthonormal system in $V(G)$. To prove $(20)$, in the following we suppress the subscript $i$ and consider the difference

$$
\Theta_{n}=\frac{n}{2}\left(\Psi \circ X^{n}-\Psi \circ X^{-n}, v^{n+1}\right)-(v \cdot \nabla \Psi, v) .
$$

Using the decomposition

$$
\begin{aligned}
\Theta_{n} & =\frac{n}{2}\left(\Psi \circ X^{n}-\Psi \circ X^{-n}, v^{n+1}-v\right)+\left(\frac{n}{2}\left[\Psi \circ X^{n}-\Psi \circ X^{-n}\right]-v \cdot \nabla \Psi, v\right) \\
& =: \alpha_{n}+\beta_{n},
\end{aligned}
$$

we find by Hölder's inequality

$$
\alpha_{n} \leq \frac{n}{2}\left\|\Psi \circ X^{n}-\Psi \circ X^{-n}\right\|\left\|v^{n+1}-v\right\|,
$$


hence, using (19), $\alpha_{n} \rightarrow 0$ as $n \rightarrow \infty$ if and only if

$$
\delta_{n}:=\frac{n}{2}\left\|\Psi \circ X^{n}-\Psi \circ X^{-n}\right\| \leq \text { const. }
$$

with some constant independent of $n$. To prove (21) we observe, setting $\varepsilon:=\frac{1}{n}$,

$$
\begin{aligned}
& \frac{n}{2}\left[\Psi \circ X^{n}-\Psi \circ X^{-n}\right]=\frac{n}{2}\left[\Psi \circ X^{n}-\Psi+\Psi-\Psi \circ X^{-n}\right] \\
& =\frac{n}{2} \int_{0}^{\varepsilon}\left[\partial_{t} X(t, \cdot) \cdot \nabla_{\mathrm{x}} \Psi(X(t, \cdot))+\partial_{t}\left(X(t-\varepsilon, \cdot) \cdot \nabla_{\mathrm{x}} \Psi(X(t-\varepsilon, \cdot))\right] \mathrm{d} t\right. \\
& =\frac{n}{2} \int_{0}^{\varepsilon}\left[\left(v^{n} \cdot \nabla_{\mathrm{x}} \Psi\right) \circ X(t, \cdot)+\left(v^{n} \cdot \nabla_{\mathrm{x}} \Psi\right) \circ X(t-\varepsilon, \cdot)\right] \mathrm{d} t,
\end{aligned}
$$

hence

$$
\begin{aligned}
\frac{n}{2}\left\|\Psi \circ X^{n}-\Psi \circ X^{-n}\right\| & \leq \max _{t \in[-\varepsilon, \varepsilon]}\left\|\left(v^{n} \cdot \nabla_{\mathrm{x}} \Psi\right) \circ X(t, \cdot)\right\|=\left\|v^{n} \cdot \nabla \Psi\right\| \\
& \leq\left\|v^{n}\right\|\|\nabla \Psi\|_{\infty} \leq C_{G, \Psi}\left\|\nabla v^{n}\right\| \leq C_{G, F, \nu, \Psi}
\end{aligned}
$$

independent of $n$ due to the boundedness of $\left(v^{n}\right)_{n}$ in $V(G)$. This proves $(21)$ and the estimate for $\alpha_{n}$. To estimate $\beta_{n}$ we decompose

$$
\beta_{n}=\left(\frac{n}{2}\left[\Psi \circ X^{n}-\Psi\right]-\frac{1}{2} v \cdot \nabla \Psi, v\right)+\left(\frac{n}{2}\left[\Psi-\Psi \circ X^{-n}\right]-\frac{1}{2} v \cdot \nabla \Psi, v\right) .
$$

Thus it suffices to consider the first term (the second analogously) in the form

$$
\theta_{n}:=\left(n\left[\Psi \circ X^{n}-\Psi\right]-v \cdot \nabla \Psi, v\right) .
$$

Setting again $\varepsilon:=\frac{1}{n}$ we obtain

$$
\begin{aligned}
\theta_{n} & =\left(n \int_{0}^{\varepsilon}\left(v^{n} \cdot \nabla_{\mathrm{x}} \Psi\right) \circ X(t, \cdot) \mathrm{d} t-v \cdot \nabla \Psi, v\right) \\
& =n \int_{0}^{\varepsilon}\left(\left(v^{n} \cdot \nabla_{\mathrm{x}} \Psi\right) \circ X(t, \cdot)-v \cdot \nabla \Psi, v\right) \mathrm{d} t \\
& =n \int_{0}^{\varepsilon}\left(\left(\left[v^{n}-v\right] \cdot \nabla_{\mathrm{x}} \Psi\right) \circ X(t, \cdot)+\left(v \cdot \nabla_{\mathrm{x}} \Psi\right) \circ X(t, \cdot)-v \cdot \nabla \Psi, v\right) \mathrm{d} t \\
& =n \int_{0}^{\varepsilon}\left(\left(\left[v^{n}-v\right] \cdot \nabla_{\mathrm{x}} \Psi\right) \circ X(t, \cdot), v\right) \mathrm{d} t-n \int_{0}^{\varepsilon}(v \cdot \nabla \Psi, v-v \circ X(-t, \cdot)) \mathrm{d} t \\
& =: \varphi_{n}-\xi_{n} .
\end{aligned}
$$

Because of (19) and the measure preserving property of the mapping $X(t, \cdot)$ we find

$$
\varphi_{n} \leq\left\|v^{n}-v\right\|\|\nabla \Psi\|_{\infty}\|v\| \rightarrow 0 \quad \text { as } \quad n \rightarrow \infty .
$$

Finally, using

$$
\begin{aligned}
& v(x)-v(X(-t, x)) \\
& =v(X(0, x))-v(X(-t, x))=\int_{-t}^{0} \partial_{s} X(s, x) \cdot \nabla_{\mathrm{x}} v(X(s, x)) \mathrm{d} s \\
& =\int_{-t}^{0} v^{n}\left(X(s, x) \cdot \nabla_{\mathrm{x}} v(X(s, x)) \mathrm{d} s=\int_{-t}^{0}\left(v^{n} \cdot \nabla_{\mathrm{x}} v\right) \circ(X(s, x)) \mathrm{d} s,\right.
\end{aligned}
$$


we conclude by Sobolev's inequality $\left(\varepsilon:=\frac{1}{n}\right)$

$$
\begin{aligned}
\xi_{n} & \leq \max _{t \in[-\varepsilon, \varepsilon]}|(v \cdot \nabla \Psi, v-v \circ X(-t, \cdot))| \\
& \leq \max _{t \in[-\varepsilon, \varepsilon]}\|v\|_{0,6}\|\nabla \Psi\|_{\infty}\left\|\int_{-t}^{0}\left(v^{n} \cdot \nabla_{\mathrm{x}} v\right) \circ(X(s, \cdot)) \mathrm{d} s\right\|_{0,6 / 5} \\
& \leq\|v\|_{0,6}\|\nabla \Psi\|_{\infty} \int_{-\varepsilon}^{0}\left\|\left(v^{n} \cdot \nabla_{\mathrm{x}} v\right) \circ(X(s, \cdot))\right\|_{0,6 / 5} \mathrm{~d} s \\
& \leq \varepsilon\|v\|_{0,6}\|\nabla \Psi\|_{\infty}\left\|v^{n} \cdot \nabla v\right\|_{0,6 / 5} \\
& \leq \varepsilon\|v\|_{0,6}\|\nabla \Psi\|_{\infty}\left\|v^{n}\right\|_{0,3}\|\nabla v\| \quad(5 / 6=1 / 3+1 / 2) \\
& \leq \varepsilon C_{G}\|\nabla \Psi\|_{\infty}\left\|\nabla v^{n}\right\|\|\nabla v\|^{2} \\
& \leq \varepsilon C_{G, F, \nu, \Psi} \rightarrow 0 \quad \text { as } \quad n \rightarrow \infty .
\end{aligned}
$$

Here for the last estimate we use $\|u\|_{s} \leq C_{G}\|\nabla u\|$, valid for all $u \in H^{1}(G)$ in bounded domains $G \subset R^{3}$ if $1 \leq s \leq 6,[1]$. This proves the theorem.

2. The non-stationary Lagrangian approximation. Let $v \in C\left(I, H^{3}(G) \cap V(G)\right)$ be given and consider for $\left(s, x_{s}\right) \in I \times G$ the non-autonomous system

$$
\dot{x}(t)=v(t, x(t)), \quad x(s)=x_{s} .
$$

Because $v$ vanishes on $I \times \partial G$ and, as $H^{3}(G)$-continuous function, certainly satisfies a uniqueness condition for (22), the solution $t \rightarrow x(t)=: X\left(t, s, x_{s}\right)$ exists in the whole interval $I$ and is uniquely determined there. Due to the uniqueness, the mappings

$$
X(t, s):=X(t, s, \cdot):\left\{\begin{array}{l}
G \rightarrow G \\
x \rightarrow X(t, s, x)
\end{array}\right.
$$

satisfy $X(t, s) \circ X(s, r):=X(t, s, X(s, r, \cdot))=X(t, r)$ for all $t, s, r \in I$, and, in particular, $X(t, s)$ is a $C^{1}$-diffeomorphism in $G$ with inverse mapping $(X(t, s))^{-1}=X(s, t)$. Since $v=0$ on $I \times \partial G$ implies $X(t, s, G)=G$, and since $\nabla \cdot v=0$ in $I \times G$, we obtain from Liouville's differential equation

$$
\partial_{t} \operatorname{det} \nabla X(t, s, x)=\nabla_{X} \cdot v(t, X(t, s, x)) \cdot \operatorname{det} \nabla X(t, s, x)=0,
$$

hence the Jacobian does not depend on $t$ :

$$
\operatorname{det} \nabla X(t, s, x)=\operatorname{det} \nabla X(s, s, x)=\operatorname{det} \nabla x=1 .
$$

As in the steady case, this volume conserving property leads to

$$
\|v(t, X(s, r, \cdot))\|_{o, p}=\|v(t, \cdot)\|_{o, p} \quad(1 \leq p \leq \infty)
$$

which holds for all $t, s, r \in I$.

In order to approximate the nonlinear convective term $v \cdot \nabla v$ of (1) we use total differences as in the stationary case:

Definition 2.1. Let $t, s, s+h \in I(h>0), x \in G$ and assume $v \in C\left(I, H^{3}(G) \cap V(G)\right)$. Let $X(\cdot, \cdot)$ denote the mapping constructed from $(22)$ and (23). Then we call the expressions

$$
\frac{1}{h}\{v(t, X(s+h, s, x))-v(t, x)\}, \quad \frac{1}{h}\{v(t, x)-v(t, X(s, s+h, x))\}
$$


one-sided Lagrangian difference quotients and

$$
\frac{1}{2 h}\{v(t, X(s+h, s, x))-v(t, X(s, s+h, x))\}
$$

a central Lagrangian difference quotient, respectively.

For $h \rightarrow 0$, every quotient in the above definition converges to $v(s, x) \cdot \nabla v(t, x)$. For instance, using (22), we obtain

$$
\begin{aligned}
v(t, X(s+h, s, x))-v(t, x) & =v(t, X(s+h, s, x))-v(t, X(s, s, x)) \\
& =\int_{s}^{s+h} \partial_{r} X(r, s, x) \cdot \nabla v(t, X(r, s, x)) \mathrm{d} r \\
& =\int_{s}^{s+h}(v(r) \cdot \nabla v(t)) \circ X(r, s, x) \mathrm{d} r,
\end{aligned}
$$

and a mean value theorem yields the assertion. But in contrast to (25), for the central quotient (26) again an $L^{2}$-orthogonality relation holds:

Lemma 2.2. Let $v \in C\left(I, H^{3}(G) \cap V(G)\right)$ be given. Then for the central Lagrangian quotient (26) we have

$$
\left(\frac{1}{2 h}[v(t, X(s+h, s, \cdot))-v(t, X(s, s+h, \cdot))], v(t, \cdot)\right)=0 .
$$

Proof. Due to $(X(q, r))^{-1}=X(r, q)$ for $q, r \in I$ and the measure preserving property of the mappings $X(q, r)$ this follows from

$$
\begin{aligned}
\left(v(t, X)-v\left(t, X^{-1}\right), v(t)\right) & \left.=(v(t, X), v(t))-v\left(t, X^{-1} \circ X\right), v(t, X)\right) \\
& =(v(t, X), v(t))-(v(t), v(t, X))=0 .
\end{aligned}
$$

The relation (27) is an analogy for the relation $(v(s) \cdot \nabla v(t), v(t))=0$, valid if $v(s) \in V(G)$, $v(t) \in H_{0}^{1}(G)$, which can be used to show the global existence of weak Navier-Stokes solutions. Thus it follows from the proof above that the central quotient leads to an energy conserving approximation.

In order to avoid fixed point considerations (both the velocity and the corresponding trajectories are not known), we additionally use a time delay and substitute the nonlinear convective term $v(t, x) \cdot \nabla v(t, x)$ by central differences

$$
\frac{1}{2 h}\{v(t, X(s+h, s, x))-v(t, X(s, s+h, x))\}
$$

assuming $s+h<t$. This leads to an approximation, where the velocity and the trajectories have to be determined from each other successively. Specifically we choose the following scheme:

Assume $T>0$ and $2 \leq N \in \mathbb{N}$. Define $h:=\frac{T}{N}>0$ and let $t_{i}:=i h(i=-2,-1, \ldots, N)$ be a grid on $[-2 h, T]$. Now for $(t, x) \in\left[t_{k}, t_{k+1}\right] \times G(k=0,1, \ldots, N-1)$ we replace $v(t, x) \cdot \nabla v(t, x)$ by

$$
\begin{aligned}
Z_{h} v(t, x):= & Z_{h}^{k} v(t, x) \\
:= & \frac{t-t_{k}}{2 h^{2}}\left[v\left(t, X\left(t_{k}, t_{k-1, x}\right)\right)-v\left(t, X\left(t_{k-1}, t_{k}, x\right)\right)\right] \\
& \quad+\frac{t_{k+1}-t}{2 h^{2}}\left[v\left(t, X\left(t_{k-1}, t_{k-2}, x\right)\right)-v\left(t, X\left(t_{k-2}, t_{k-1}, x\right)\right)\right] .
\end{aligned}
$$


REMARK 2.3. (a) The determination of $v(t)$ for $t \in\left[t_{0}, t_{1}\right]$ requires a special initial construction, which is carried out in the next section.

(b) In (28) the mappings $X: G \rightarrow G$ do not depend on $t \in\left[t_{k}, t_{k+1}\right]$, which means a simplification from the numerical point of view, since we only have to compute four mappings $X(\cdot, \cdot)$ in $\left[t_{k}, t_{k+1}\right]$. Nevertheless, the continuity on $[0, T]$ of the functions $Z_{h} v(\cdot, x)$ does still hold and ensures the global existence of a unique solution in the next section.

3. Global existence, uniqueness, compatibility. It is known ([9], [13], [17]) that the compatibility condition, which has to be satisfied by any solution of the non-stationary Navier-Stokes equations (1) in case of strong $H^{3}$-continuity at $t=0$, cannot be proved in general, if the corresponding initial velocity $v_{0}$ is given. But still, following a hint of Solonnikov [25], we can construct an initial velocity $v_{0}$ in such a way, that this condition is fulfilled, and in the present case of scheme (28), moreover, this construction is unique. To do so, for simplicity we assume conservative external forces $(F=0)$ and a kinematic viscosity normalized to one $(\nu=1)$ in the Navier-Stokes equations (1). Now replacing the convective term by (28) and the initial condition $v(0)=v_{0}$ by $\partial_{t} v(0)=a_{0}$, we obtain at time $t=0$ in $G$ the stationary (projected) equations

$$
P\left(a_{0}-\Delta v_{0}+\frac{1}{2 h}\left[v_{0} \circ X(-h,-2 h)-v_{0} \circ X(-2 h,-h)\right]\right)=0
$$

with the prescribed initial acceleration $a_{0}$. The construction of the initial velocity is now stated in

Lemma 3.1. Assume $T>0, u \in C\left([-T, 0], H^{3}(G) \cap V(G)\right)$ and $a_{0} \in V(G)$. Let $2 \leq N \in$ $\mathbb{N}$ and define $h:=\frac{T}{N}>0$. Then:

(a) Replacing $v$ by $u$ in (22), the mappings $X(-h,-2 h)$ and $X(-2 h,-h)$ in (29) are uniquely defined by (23).

(b) There exists a uniquely determined solution $v_{0} \in H^{3}(G) \cap V(G)$ of (29). The function $v_{0}$ satisfies the estimate

$$
\left\|\nabla v_{0}\right\| \leq c\left\|a_{0}\right\|
$$

with the Poincaré constant c.

(c) The function $v$ given by

$$
v(t):= \begin{cases}u(t) & \text { for } t \in[-T,-h], \\ \frac{1}{h}\left\{(t+h) v_{0}-t u(-h)\right\} & \text { for } t \in[-h, 0],\end{cases}
$$

belongs to $C\left([-T, 0], H^{3}(G) \cap V(G)\right)$, and hence the mappings $X(0,-h)$ and $X(-h, 0)$ in (28) are uniquely defined by (22), (23).

Proof. Because (a) and (c) are obvious, we only have to show (b). To prove uniqueness let $w_{0}:=v_{0}^{1}-v_{0}^{2}$ be the difference of two solutions $v_{0}^{1}, v_{0}^{2}$. With $X:=X(-h,-2 h)$ we thus obtain

$$
P\left(-\Delta w_{0}+\frac{1}{2 h}\left[w_{0} \circ X-w_{0} \circ X^{-1}\right]\right)=0 .
$$

Because of $\left(w_{0} \circ X-w_{0} \circ X^{-1}, w_{0}\right)=0$ the uniqueness then follows by Poincaré's inequality

$$
\left\|w_{0}\right\| \leq c_{G}\left\|\nabla w_{0}\right\|=0
$$


The existence of a solution $v_{0} \in V(G)$ can be shown in the same way as for the nonlinear stationary Navier-Stokes equations [16], and concerning the regularity statement $v_{0} \in$ $H^{3}(G)$, due to

$$
\left\|v_{0}\right\|_{3} \leq c\left(\left\|a_{0}\right\|_{1}+\left\|v_{0} \circ X\right\|_{1}+\left\|v_{0} \circ X^{-1}\right\|_{1}\right)
$$

by Cattabriga's estimate [3], it remains to prove $\left\|v_{0} \circ Y\right\|_{1}<\infty$ for $Y \in\left\{X, X^{-1}\right\}$. To do so, we first observe, that $\|\nabla Y\|_{\infty}<\infty$ because of the strong $H^{3}(G)$-continuity of $u$. Hence everything is shown due to

$$
\left\|v_{0} \circ Y\right\|=\left\|v_{0}\right\|,\left\|\nabla\left(v_{0} \circ Y\right)\right\| \leq\left\|\left(\nabla_{Y} v_{0}\right) \circ Y\right\|\|\nabla Y\|_{\infty}=\left\|\nabla v_{0}\right\|\|\nabla Y\|_{\infty} .
$$

Theorem 3.2. Let $T>0, u \in C\left([-T, 0], H^{3}(G) \cap V(G)\right)$ and $a_{0} \in V(G)$ be given. Let $2 \leq N \in \mathbb{N}$ and define $h:=\frac{T}{N}>0$. Assume that the initial construction is carried out as in Lemma 3.1, and that, in particular, $v_{0} \in H^{3}(G) \cap V(G)$ denotes the unique solution of (29). Then there exist unique functions $v \in C^{l}\left([0, T], H^{3-2 l}(G) \cap V(G)\right), l \in\{0,1\}$, and $\nabla p \in C\left([0, T], H^{1}(G)\right)$ satisfying

$$
\begin{aligned}
\partial_{t} v-\Delta v+Z_{h} v+\nabla p & =0 & & \text { in } G_{T}, \\
\nabla \cdot v & =0 & & \text { in } G_{T}, \\
v & =0 & & \text { on } \partial G, \\
v & =v_{0} & & \text { for } t=0,
\end{aligned}
$$

where $Z_{h} v$ is defined by (28). For $t \in[0, T]$ the function $v$ satisfies the energy equation

$$
\|v(t)\|^{2}+2 \int_{0}^{t}\|\nabla v(s)\|^{2} \mathrm{~d} s=\left\|v_{0}\right\|^{2} .
$$

Proof. Let us assume $t \in[0, h]$. Here the system (33) is linear, and as in [8] a Galerkin method shows the existence of uniquely determined functions $v^{0} \in C\left([0, h], H^{2}(G) \cap\right.$ $V(G))$ with $\partial_{t} v^{0} \in C([0, h], H(G))$ and $\nabla p^{0} \in C\left([0, h], L^{2}(G)\right)$ satisfying (33) and (34) on $[0, h]$. Now applying a result of Temam [17] it follows that on $[0, h]$ the functions $v^{0}, \nabla p^{0}$ possess all the continuity properties, which in Theorem 3.2 are asserted to hold on $[0, T]$. This is due to $v_{0} \in H^{3}(G) \cap V(G)$ and the fact that, because of $\partial_{t} v(0)=a_{0} \in V(G)$, the compatibility condition

$$
P\left(-\Delta v_{0}+\frac{1}{2 h}\left[v_{0} \circ X(-h,-2 h)-v_{0} \circ X(-2 h,-h)\right]\right)=0 \text { on } \partial G
$$

is forced to be satisfied. Hence using $v:=v^{0}$ in $(22)$, the mappings $X(h, 0)$ and $X(0, h)$ are uniquely defined by $(23)$, and we are ready to continue the procedure on the next subinterval.

To do so, set $v_{1}:=v^{0}(h) \in H^{3}(G) \cap V(G)$, replace $v(0)=v_{0}$ by $v(h)=v_{1}$ in $(33)$, and consider the resulting equations for $t \in[h, 2 h]$, only. Here again, these equations are linear, and the unique solution $v^{1}, \nabla p^{1}$ on $[h, 2 h]$ has the same degree of regularity as $v^{0}, \nabla p^{0}$ on $[0, h]$. To see this, we observe that $P \Delta v_{1}=P \Delta v^{0}(h)$ and $Z_{h}^{1} v_{1}=Z_{h}^{0} v^{0}(h)$ by (28), hence $\partial_{t} v^{1}(h)=\partial_{t} v^{0}(h) \in V(G)$, and therefore again the corresponding compatibility condition

$$
P\left(-\Delta v_{1}+\frac{1}{2 h}\left\{v_{1} \circ X(0,-h)-v_{1} \circ X(-h, 0)\right\}\right)=0 \text { on } \partial G
$$

is satisfied. Thus, repeating this procedure on all subintervals, the theorem is proved. 
REMARK 3.3. The global construction in the proof above works without any smallness assumptions for the prescribed initial acceleration $a_{0}$ and the function $u$ in Lemma 3.1. Due to appearing nonlinearities, a similar construction to satisfy higher order compatibility conditions (cf. Temam [17]) without any smallness assumptions does not seem to be possible up to now.

4. Construction of weak solutions. In the general three dimensional case the only solutions of the Navier-Stokes equations (1), whose existence for all time has been proved, are solutions in a weak sense (Hopf [10]; compare also Temam [16]). Let us recall:

Definition 4.1. Assume $T>0$ and $v_{0} \in H(G)$. Then a function $v \in L^{2}(0, T, V(G)) \cap$ $L^{\infty}(0, T, H(G))$ is called a weak solution of the Navier-Stokes equations (1) with $F=0$, $\nu=1$ and initial value $v_{0}$, if $v:[0, T] \rightarrow H(G)$ is weakly continuous, if $\left\|v(t)-v_{0}\right\| \rightarrow 0$ for $t \rightarrow 0$, and if for all $\Phi \in C_{0}^{\infty}((0, T) \times G)$ with $\Phi(t) \in D(0<t<T)$ the identity

$$
\int_{0}^{T}\left[-\left(v(t), \partial_{t} \Phi(t)\right)+(\nabla v(t), \nabla \Phi(t))-(v(t) \cdot \nabla \Phi(t), v(t))\right] \mathrm{d} t=0
$$

is satisfied.

We show that such a solution can be constructed from the solution of the system (33), if in Theorem 3.2 for $N \rightarrow \infty$ ( $T$ remains fixed) the step size $h:=\frac{T}{N}>0$ goes to zero. To express the dependence on $N$, in the following we write $h_{N}, v^{N}, v_{0}^{N}$ instead of $h, v, v_{0}$. A main result is now stated in

TheOrem 4.2. Let $T>0$ be fixed, and let $h_{N}:=\frac{T}{N}>0$ for $2 \leq N \in \mathbb{N}$. As constructed in Lemma 3.1 and Theorem 3.2, respectively, let $v_{0}^{N}$ and $v^{N}$ denote the initial value and the solution of the corresponding equations (33). Then there exists a convergent subsequence $\left(v_{0}^{N_{k}}\right)_{k}$ of $\left(v_{0}^{N}\right)_{N}$ with limit $v_{0}$ and a convergent subsequence $\left(v^{N_{k}}\right)_{k}$ of $\left(v^{N}\right)_{N}$ with limit $v$ such that $v$ is a weak solution of the Navier-Stokes equations (1) with $F=0, \nu=1$ and initial value $v_{0}$. The function $v$ satisfies for $t \in[0, T]$ the energy inequality

$$
\|v(t)\|^{2}+2 \int_{0}^{t}\|\nabla v(s)\|^{2} \mathrm{~d} s \leq\left\|v_{0}\right\|^{2} .
$$

Proof. (a) First let us derive some estimates independent of $N$. To do so, in the following we denote by $c, c_{1}, c_{2}, \ldots$ generic constants, which do not depend on $n$. By (34), Poincaré's inequality and (30) it follows for all $t \in[0, T]$

$$
\left\|v^{N}(t)\right\|^{2}+2 \int_{0}^{t}\left\|\nabla v^{N}(s)\right\|^{2} \mathrm{~d} s=\left\|v_{0}^{N}\right\|^{2} \leq c\left\|\nabla v_{0}^{N}\right\|^{2} \leq c_{1},
$$

and thus for all $t \in[-T, 0]$ by $(31)$

$$
\left\|v^{N}(t)\right\|^{2} \leq c\left\|\nabla v^{N}(t)\right\|^{2} \leq c_{2} .
$$

Both constants do not depend on $N$ and $t$. Now let $B:=\left\{e_{i} \mid i \in \mathbb{N}\right\} \subset D$ denote a complete orthonormal system in $H(G)$. Next we show that

$$
\left|\left(\partial_{t} v^{N}(t), e_{i}\right)\right| \leq c_{i}
$$


for every $i \in \mathbb{N}$. Here the constant depends on the basis function $e_{i}$, but not on $N$ and $t \in[0, T]$. To do so, let $e:=e_{i}$ and $h:=h_{N}$ for brevity of notation. Because of

$$
\left(\Delta v^{N}(t), e\right)=\left(v^{N}(t), \Delta e\right), \quad\left(Z_{h} v^{N}(t), e\right)=-\left(\left(Z_{h} e\right)(t), v^{N}(t)\right)
$$

using (24) for $p=2$, it follows by (37) that (39) is proved if

$$
\left\|\left(Z_{h} e\right)(t)\right\| \leq c
$$

with a constant independent of $N$ and $t \in[0, T]$. Here for $t \in\left[t_{k}, t_{k+1}\right](k=0,1, \ldots, N-1)$ the term $\left(Z_{h} e\right)(t)$ is defined by

$$
\left(Z_{h} e\right)(t):=\frac{t-t_{k}}{2 h^{2}}\left\{e \circ X_{k}-e \circ X_{k}^{-1}\right\}+\frac{t_{k+1}-t}{2 h^{2}}\left\{e \circ X_{k-1}-e \circ X_{k-1}^{-1}\right\}
$$

with

$$
X_{l}:=X\left(t_{l}, t_{l-l}\right), X_{l}^{-1}:=X\left(t_{l-1}, t_{l}\right)
$$

for $l=-1,0, \ldots, N-1$. Now using (22), (23) to obtain

$$
\begin{aligned}
e \circ X_{k}-e \circ X_{k}^{-1}= & e \circ X_{k}-e+e-e \circ X_{k}^{-1} \\
= & \int_{t_{k-1}}^{t_{k}}\left\{\partial_{s} X\left(s, t_{k-1}\right) \cdot \nabla_{X} e \circ X\left(s, t_{k-1}\right)\right. \\
& \left.+\partial_{s} X\left(s, t_{k}\right) \cdot \nabla_{X} e \circ X\left(s, t_{k}\right)\right\} \mathrm{d} s \\
= & \int_{t_{k-1}}^{t_{k}}\left\{\left(v^{N}(s) \cdot \nabla e\right) \circ X\left(s, t_{k-1}\right)+\left(v^{N}(s) \cdot \nabla e\right) \circ X\left(s, t_{k}\right)\right\} \mathrm{d} s,
\end{aligned}
$$

which by (24), (37) and (38) implies

$$
\left\|e \circ X_{k}-e \circ X_{k}^{-1}\right\| \leq 2 h \max _{s_{1}, s_{2}}\left\|\left(v^{N}\left(s_{1}\right) \cdot \nabla e\right) \circ X\left(s_{1}, s_{2}\right)\right\| \leq h c,
$$

and the estimate (40) is proved.

(b) Due to (37) and (39) it follows as in [10] that there exists (after possibly redefinition on a set of measure zero) a weakly continuous function $v:[0, T] \rightarrow H(G)$ with $v \in$ $L^{2}(0, T, V(G)) \cap L^{\infty}(0, T, H(G))$ and a subsequence $\left(v^{N_{k}}\right)_{k}$ of $\left(v^{N}\right)_{N}$ such that for $k \rightarrow \infty$

$$
\begin{aligned}
v^{N_{k}}(t) \rightarrow v(t) & \text { weakly in } H(G) \quad(t \in[0, T]), \\
v^{N_{k}} \rightarrow v & \text { weakly in } L^{2}(0, T, V(G)), \\
v^{N_{k}} \rightarrow v & \text { strongly in } L^{2}(0, T, H(G)) .
\end{aligned}
$$

Moreover, by (37) and the compactness of the imbedding $V(G) \rightarrow H(G)$ there exists a function $v_{0} \in V(G) \subset H(G)$ with

$$
v_{0}^{N_{k}} \rightarrow v_{0} \quad \text { strongly in } H(G)
$$

as $k \rightarrow \infty$. Hence by

$$
\begin{aligned}
\|v(t)\|^{2}+2 \int_{0}^{t}\|\nabla v(s)\|^{2} \mathrm{~d} s & \leq \liminf _{k \rightarrow \infty}\left(\left\|v^{N_{k}}(t)\right\|^{2}+2 \int_{0}^{t}\left\|\nabla v^{N_{k}}(s)\right\|^{2} \mathrm{~d} s\right) \\
& \leq \lim _{k \rightarrow \infty}\left\|v_{0}^{N_{k}}-v_{0}+v_{0}\right\|^{2}=\left\|v_{0}\right\|^{2}
\end{aligned}
$$

the function $v$ satisfies (36) for $t \in[0, T]$, and thus $v(t) \rightarrow v_{0}$ strongly in $H(G)$ as $t \rightarrow 0$. It remains to show (35), where we may restrict us to functions $\Phi_{i}=\varphi e_{i}$ with a scalar 
function $\varphi \in C_{0}^{\infty}((0, T))$ and $e_{i} \in B$. Now using (43) and (44) the theorem is proved if

$$
\lim _{k \rightarrow \infty} \int_{0}^{T}\left(\left(Z_{h_{N_{k}}} e_{i}\right)(t), v^{N_{k}}(t)\right) \mathrm{d} t=\int_{0}^{T}\left(v(t) \cdot \nabla e_{i}, v(t)\right) \mathrm{d} t
$$

holds for every $i \in \mathbb{N}$.

(c) Without loss of generality let us assume the convergence of the whole sequence in the corresponding norms above and write

$$
\lim _{N \rightarrow \infty} \int_{0}^{T}\left\{\left(\left(Z_{h} e\right)(t), v^{N}(t)\right)-(v(t) \cdot \nabla e, v(t))\right\} \mathrm{d} t=0
$$

instead of (46), for simplicity. For the integrand $I^{N}(t)$ in (47) we have

$$
I^{N}(t)=\left(\left(Z_{h} e\right)(t), v^{N}(t)-v(t)\right)+\left(\left(Z_{h} e\right)(t)-v(t) \cdot \nabla e, v(t)\right)=: P_{1}^{N}(t)+P_{2}^{N}(t),
$$

where

$$
\int_{0}^{T} P_{1}^{N}(t) \mathrm{d} t \rightarrow 0 \text { as } N \rightarrow \infty
$$

because of (40) and (44). Due to (41) for the second term we obtain

$$
\begin{aligned}
P_{2}^{N}(t)= & \frac{t-t_{k}}{2 h}\left(\frac{1}{h}\left\{e \circ X_{k}-e\right\}-v(t) \cdot \nabla e, v(t)\right) \\
& +\frac{t-t_{k}}{2 h}\left(\frac{1}{h}\left\{e-e \circ X_{k}^{-1}\right\}-v(t) \cdot \nabla e, v(t)\right) \\
& +\frac{t_{k+1}-t}{2 h}\left(\frac{1}{h}\left\{e \circ X_{k-1}-e\right\}-v(t) \cdot \nabla e, v(t)\right) \\
& +\frac{t_{k+1}-t}{2 h}\left(\frac{1}{h}\left\{e-e \circ X_{k-1}^{-1}\right\}-v(t) \cdot \nabla e, v(t)\right) \\
= & : \sum_{j=1}^{4} Q_{j}^{N}(t) .
\end{aligned}
$$

Because all terms $Q_{j}^{N}$ can be treated in the same way, we only consider $Q_{1}^{N}$. By (22) and (23), using $X:=X\left(s, t_{k-1}\right)$ for abbreviation, we find

$$
\begin{aligned}
Q_{1}^{N}(t)= & \frac{t-t_{k}}{2 h}\left(\frac{1}{h} \int_{t_{k-1}}^{t_{k}} \partial_{s} X \cdot \nabla_{X} e \circ X \mathrm{~d} s-v(t) \cdot \nabla e, v(t)\right) \\
= & \frac{t-t_{k}}{2 h^{2}} \int_{t_{k-1}}^{t_{k}}\left(\left(v^{N}(s) \cdot \nabla_{X} e\right) \circ X-v(t) \cdot \nabla e, v(t)\right) \mathrm{d} s \\
= & \frac{t-t_{k}}{2 h^{2}} \int_{t_{k-1}}^{t_{k}}\left(\left(v^{N}(s)-v^{N}(t)\right) \cdot \nabla e, v(t) \circ X^{-1}\right) \mathrm{d} s \\
& +\frac{t-t_{k}}{2 h^{2}} \int_{t_{k-1}}^{t_{k}}\left(\left(v^{N}(t)-v(t)\right) \cdot \nabla e, v(t) \circ X^{-1}\right) \mathrm{d} s \\
& -\frac{t-t_{k}}{2 h^{2}} \int_{t_{k-1}}^{t_{k}}\left(v(t) \cdot \nabla e, v(t)-v(t) \circ X^{-1}\right) \mathrm{d} s \\
= & \sum_{j=1}^{3} R_{j}^{N}(t) .
\end{aligned}
$$


Thus (47) holds if

$$
\lim _{N \rightarrow \infty}\left|\int_{0}^{T} R_{j}^{N}(t) \mathrm{d} t\right|=0 \quad(j=1,2,3)
$$

is proved.

(d) In the following estimates, all constants $c, c_{1}, c_{2}, \ldots$ are independent of $N$. Due to (36) we have

$$
\begin{aligned}
\left|\int_{0}^{T} R_{1}^{N}(t) \mathrm{d} t\right| & =\left|\sum_{k=0}^{N-1} \int_{t_{k}}^{t_{k+1}} R_{1}^{N}(t) \mathrm{d} t\right| \\
& \leq \frac{1}{2 h} \sum_{k=0}^{N-1} \int_{t_{k}}^{t_{k+1}} \int_{t_{k-1}}^{t_{k}}\left\|v^{N}(s)-v^{N}(t)\right\|\|\nabla e\|_{\infty}\|v(t)\| \mathrm{d} s \mathrm{~d} t \\
& \leq \frac{c_{1}}{h} \sum_{k=0}^{N-1} \int_{t_{k}}^{t_{k+1}} \int_{t_{k-1}}^{t_{k}}\left\|v^{N}(s)-v^{N}(t)\right\| \mathrm{d} s \mathrm{~d} t .
\end{aligned}
$$

Since $s \leq 0$ for $k=0$, the first summand will be treated separately, to obtain

$$
\frac{c_{1}}{h} \int_{0}^{h} \int_{-h}^{0}\left\|v^{N}(s)-v^{N}(t)\right\| \mathrm{d} s \mathrm{~d} t \leq \frac{c_{1}}{h} \int_{0}^{h} \int_{-h}^{0}\left\{\left\|v^{N}(s)\right\|+\left\|v^{N}(t)\right\|\right\} \mathrm{d} s \mathrm{~d} t \leq \frac{c}{N}
$$

due to (37) and (38). Using the Friedrichs inequality it follows that for every $\delta>0$ there exists a number $M_{\delta} \in \mathbb{N}$ with

$$
\begin{aligned}
\left\|v^{N}(s)-v^{N}(t)\right\| & \leq \sum_{j=1}^{M_{\delta}}\left|\left(v^{N}(s)-v^{N}(t), e_{j}\right)\right|+\delta\left\{\left\|\nabla v^{N}(s)\right\|+\left\|\nabla v^{N}(t)\right\|\right\} \\
& =: g_{1}^{N}(s, t)+\delta g_{2}^{N}(s, t) .
\end{aligned}
$$

Since $s, t \geq 0$ we have by $(39)\left|\left(v^{N}(s)-v^{N}(t), e_{j}\right)\right| \leq c_{j}|s-t| \leq 2 c_{j} h$, and thus

$$
\frac{c_{1}}{h} \sum_{k=1}^{N-1} \int_{t_{k}}^{t_{k+1}} \int_{t_{k-1}}^{t_{k}} g_{1}^{N}(s, t) \mathrm{d} s \mathrm{~d} t \leq \frac{c_{1}}{h} N h^{2}\left(\sum_{j=1}^{M_{\delta}} c_{j}\right) 2 h \leq \frac{c_{\delta}}{N} .
$$

On the other hand we obtain

$$
\begin{aligned}
\frac{c_{1}}{h} \sum_{k=1}^{N-1} \int_{t_{k}}^{t_{k+1}} \int_{t_{k-1}}^{t_{k}} g_{2}^{N}(s, t) \mathrm{d} s \mathrm{~d} t & \leq c_{1} \sum_{k=1}^{N-1}\left\{\int_{t_{k}}^{t_{k+1}}\left\|\nabla v^{N}(t)\right\| \mathrm{d} t+\int_{t_{k-1}}^{t_{k}}\left\|\nabla v^{N}(s)\right\| \mathrm{d} s\right\} \\
& \leq 2 c_{1} \int_{0}^{T}\left\|\nabla v^{N}(t)\right\| \mathrm{d} t \leq c_{2}\left(\int_{0}^{T}\left\|\nabla v^{N}(t)\right\|^{2} \mathrm{~d} t\right)^{\frac{1}{2}} \leq c_{3}
\end{aligned}
$$

by (37), and, choosing $\delta$ sufficiently small, (48) for $j=1$ is proved. The case $j=2$ follows by (36) and (44):

$$
\left|\int_{0}^{T} R_{2}^{N}(t) \mathrm{d} t\right| \leq \frac{1}{2} \int_{0}^{T}\left\|v^{N}(t)-v(t)\right\|\|\nabla e\|_{\infty}\|v(t)\| d \leq c\left(\int_{0}^{T}\left\|v^{N}(t)-v(t)\right\|^{2} \mathrm{~d} t\right)^{\frac{1}{2}} .
$$

To prove (48) for $j=3$ let $s \in\left[t_{k-1}, t_{k}\right]$. Due to

$$
v(t)-v(t) \circ X\left(t_{k-1}, s\right)=v(t) \circ X(s, s)-v(t) \circ X\left(t_{k-1}, s\right)=\int_{t_{k-1}}^{s}\left(v^{N}(r) \cdot \nabla v(t)\right) \circ X(r, s) \mathrm{d} r
$$


we obtain by Hölder's inequality and well known [1] Sobolev imbeddings

$$
\begin{aligned}
\left|R_{3}^{N}(t)\right| & \leq \frac{1}{2 h} \int_{t_{k-1}}^{t_{k}} \int_{t_{k-1}}^{s} \mid\left(\left(v(t) \cdot \nabla e,\left(v^{N}(r) \cdot \nabla v(t)\right) \circ X(r, s)\right) \mid \mathrm{d} r \mathrm{~d} s\right. \\
& \leq \frac{1}{2 h} \int_{t_{k-1}}^{s}\|v(t)\|_{0,6}\|\nabla e\|_{\infty}\left\|v^{N}(r)\right\|_{0,3}\|\nabla v(t)\| \mathrm{d} r \mathrm{~d} s \\
& \leq c_{1}\|\nabla v(t)\|^{2} \int_{t_{k-1}}^{t_{k}}\left\|\nabla v^{N}(r)\right\| \mathrm{d} r \leq c_{1}\|\nabla v(t)\|^{2} h^{\frac{1}{2}}\left(\int_{t_{k}-1}^{t_{k}}\left\|\nabla v^{N}(r)\right\|^{2} \mathrm{~d} r\right)^{\frac{1}{2}} \\
& \leq c_{1}\|\nabla v(t)\|^{2} h^{\frac{1}{2}}\left(\int_{-T}^{+T}\left\|\nabla v^{N}(r)\right\|^{2} \mathrm{~d} r\right)^{\frac{1}{2}} \leq c_{2} N^{-\frac{1}{2}}\|\nabla v(t)\|^{2}
\end{aligned}
$$

for almost all $t \in[0, T]$. Here the last estimate follows from (37) and (38). Hence

$$
\left|\int_{0}^{T} R_{3}^{N}(t) \mathrm{d} t\right| \leq c_{2} N^{-\frac{1}{2}} \int_{0}^{T}\|\nabla v(t)\|^{2} \mathrm{~d} t \leq c_{3} N^{-\frac{1}{2}}
$$

by (36), and the theorem is proved.

Choosing $u=0$ in Lemma 3.1, the system (29) reduces to the Stokes equations. Because its unique solution $v_{0}$ does not depend on $N$, in Theorem 4.2 we have $v_{0}^{N}=v_{0}$ for all $N \in \mathbb{N}(N \geq 2)$. Let us conclude with a final consideration concerning strong solutions of (1):

REMARK 4.3. In Theorem 4.2 for all $2 \leq N \in \mathbb{N}$ the same function $u$ is used for the initial construction in Lemma 3.1. The statement of Theorem 4.2 remains valid if the function $u$ depends on $N$ as follows: Let $u:=u^{N}$ be given for some $N \geq 2$. Then define $v^{N}$ by (31) and choose $u^{N+1}:=v^{N}$ in the next step. Now, under this modification, let $v_{0}$ be any accumulation point of the sequence $\left(v_{0}^{N}\right)_{N}$ mentioned in Theorem 4.2, and let $v$ be the corresponding unique strong solution of (1), existing on a (possibly small) time interval $\left[0, T^{*}\right], 0<T^{*} \leq T([6],[8],[15])$. Then it can be shown by the same methods as in the proof above that $v$ belongs to $C^{1}\left(\left[0, T^{*}\right], H^{m-2 l}(G) \cap V(G)\right), l \in\{0,1\}$.

\section{References}

[1] R. A. Adams and J. J. F. Fournier, Sobolev Spaces, Academic Press, New York, 2003.

[2] V. Arnold, Méthodes Mathématiques de la Mécanique Classique, Editions Mir, Moscow, 1976.

[3] L. Cattabriga, Su un problema al contorno relativo al sistema di equazioni di Stokes, Rend. Sem. Math. Univ. Padova 31 (1960), 308-340.

[4] D. Ebin and J. Marsden, Groups of diffeomorphisms and the motion of an incompressible fluid, Ann. of Math. 92 (1970), 102-163.

[5] C. Foias, C. Guillopé and R. Temam, Lagrangian representation of a flow, J. Diff. Equa. 57 (1985), 440-449.

[6] H. Fujita and T. Kato, On the Navier-Stokes initial value problem I, Arch. Rat. Mech. Anal. 16 (1964), 269-315.

[7] F. K. Hebeker, An approximation method for the Cauchy problem to the three-dimensional equation of vorticity transport, Math. Meth. in the Appl. Sci. 5 (1983), 439-475. 
[8] J. G. Heywood, The Navier-Stokes equations: On the existence, regularity and decay of solutions, Ind. Univ. Math. J. 29 (1980), 639-681.

[9] J. G. Heywood and R. Rannacher, Finite element approximation of the nonstationary Navier-Stokes problem. I. Regularity of solutions and second-order error estimates for spatial discretization, SIAM J. Numer. Anal. 19 (1982), 275-311.

[10] E. Hopf, Über die Anfangswertaufgabe für die hydrodynamischen Grundgleichungen, Math. Nachr. 4 (1951), 213-231.

[11] O. A. Ladyzhenskaya, The Mathematical Theory of Viscous Incompressible Flow, Gordon and Breach, New York, 1969.

[12] R. Rautmann, Ein Näherungsverfahren für spezielle parabolische Anfangswertaufgaben mit Operatoren, in: Numerische Lösungen nichtlinearer partieller Differential- und Integrodifferentialgleichungen, R. Ansorge and W. Törnig (eds.), Lecture Notes in Mathematics 267, Springer, Berlin, 11972, 81-225.

[13] R. Rautmann, On optimum regularity of Navier-Stokes solutions at time $t=0$, Math. Zeitschr. 184 (1983), 141-159.

[14] R. Rautmann and W. Varnhorn, Die Navier-Stokessche Anfangsrandwertaufgabe mit einer Differenzennäherung, Zeitschr. f. Angew. Math. u. Mech. 65 (1985), T360-T362.

[15] H. Sohr, Optimale lokale Existenzsätze für die Gleichungen von Navier-Stokes, Math. Ann. 267 (1984), 107-123.

[16] R. Temam, Navier-Stokes Equations, North-Holland, Amsterdam, 1977.

[17] R. Temam, Behaviour at time $t=0$ of the solutions of semilinear evolution equations, MRC Technical Summary Report 2162 Madison, Univ. of Wisconsin, 1980.

[18] R. Temam, Navier-Stokes Equations and Nonlinear Functional Analysis, CBMS-NSF Regional Conference Series in Applied Mathematics, SIAM, Philadelphia, 1983.

[19] P. Constantin, An Eulerian-Lagrangian approach for incompressible fluids: Local theory, J. Amer. Math. Soc. 14 (2001), 263-278.

[20] P. Constantin, An Eulerian-Lagrangian approach to the Navier-Stokes equations, Comm. Math. Phys. 216 (2001), 663-686.

[21] M. D. Gunzburger, Global existence of weak solutions for viscous incompressible flows around a moving rigid body in three dimensions, J. Math. Fluid Mech. 2 (2000), 219-266.

[22] P. L. Lions, Mathematical Topics in Fluid Mechanics 1, Oxford Universiy Press, 1996.

[23] K. Ohkitani and P. Constantin, Numerical study of the Eulerian-Lagrangian formulation of the Navier-Stokes equations, Phys. Fluids 15 (2003), 3251-3254.

[24] O. Pironneau, The method of characteristics with gradients and integrals, in: Proc. Euro Days 2000, J. Periaux (ed.), Wiley, 2001.

[25] V. A. Solonnikov, personal communication. 